\title{
Modelling PAH curvature in laminar premixed flames using a detailed population balance model
}

\author{
Edward K. Y. Yapp ${ }^{\mathrm{a}}$, Clive G. Wells ${ }^{\mathrm{b}, \mathrm{c}}$, Jethro Akroyd ${ }^{\mathrm{a}}$, \\ Sebastian Mosbach ${ }^{\mathrm{a}}$, Rong $\mathrm{Xu}^{\mathrm{d}}$, Markus Kraft ${ }^{\mathrm{a}, \mathrm{d}, *}$ \\ ${ }^{a}$ Department of Chemical Engineering and Biotechnology, University of Cambridge, \\ New Museums Site, Pembroke Street, Cambridge, CB2 3RA, United Kingdom \\ ${ }^{b}$ Centre for Mathematical Sciences, Wilberforce Road, Cambridge, CB3 0WA, \\ United Kingdom \\ ${ }^{c}$ Hughes Hall, Cambridge, CB1 2EW, United Kingdom \\ ${ }^{d}$ School of Chemical and Biomedical Engineering, \\ Nanyang Technological University, 62 Nanyang Drive, Singapore 637459
}

\begin{abstract}
A detailed population balance model, which includes the kinetic Monte Carloaromatic site (KMC-ARS) model for detailed polycyclic aromatic hydrocarbon (PAH) growth, is used to compute the Gauss curvature of PAHs in laminar premixed ethylene and benzene flames. Previous studies have found that capping of an embedded 5-member ring causes curvature in graphene edges. In this work, a capping process is added to the KMC-ARS model with the rate coefficient of the capping reaction taken from the work of You et al. [Proc. Combust. Inst., 33:685-692, 2011]. We demonstrate that the GaussBonnet theorem can be used to derive a correlation between the number of 5- and 6-member rings in a PAH and its Gauss curvature (or radius of curvature), independent of where the 5-member ring is embedded within the PAH structure. Numerical simulation yields satisfactory results when compared to the experimentally determined Gauss curvature reported in the literature. Computed and experimental fringe length distributions are also compared and the results suggest that PAHs smaller than the size required for inception are able to condense onto particles.
\end{abstract}

\footnotetext{
*Corresponding author. Tel.: +44 1223 762784; fax: +44 1223334796.

Email address: mk306@cam.ac.uk (Markus Kraft)

URL: http://como.cheng.cam.ac.uk (Markus Kraft)
} 
Keywords: Soot, PAH curvature, Premixed flame, Modeling, Gauss-Bonnet, Fringe length

\section{Introduction}

The buckminsterfullerene $\left(\mathrm{C}_{60}\right)$ was first discovered during the condensation of carbon vapour after the laser bombardment of graphite [1]; subsequently, $\mathrm{C}_{60}$ and other fullerenes were discovered in flames [2]. Fullerenes are a class of 3-D closed shells which contain exactly 12 pentagons. Their shape and size depends on the arrangement of pentagons as well as the number of hexagons. One can also consider them to be 2-D as the carbon atoms only form a molecular sheet [3]. Sheets containing less than 12 pentagons are open shells with the limiting case being a flat sheet. In a comment on the role of fullerenes in soot formation in flames, Frenklach and Ebert [4] argue that polycyclic aromatic hydrocarbons (PAHs) can curve through the incorporation of 5-member rings and can grow through sequential buildup of benzene and acenaphthylene to form fullerenes. They further argue that partially closed carbon clusters may collide and stick together while open shells continue to grow forming layers around the adducts; an explanation that is consistent with experimental high-resolution transmission electron microscopy (HRTEM) images of soot collected from flames which show multiple centres surrounded by concentric circles (see, e.g., ref. [5-9]). Quantitative measurements of these sheet-like PAHs otherwise referred to as lattice fringes have been made including measurements such as the fringe length, interlayer spacing and, more rarely, radius of curvature $[5,6]$.

To model the 3-D evolution of PAH structures in different flame environments, Violi et al. [10-12] employ a fully-coupled kinetic Monte Carlomolecular dynamics (KMC-MD) code which has a detailed PAH growth mechanism. Frenklach and co-workers [13-15] use a similar model which includes many more reactions creating 5- and 6-member complexes and more recently has been extended to include oxidation reactions [16]. However, these simulations are computationally very expensive. Therefore, these simulations are limited to the tracking of a single, non-interacting PAH; whereas experimental HRTEMs show that soot particles are made up of multiple layers of stacked PAHs. Kraft and co-workers employ a detailed population balance model [17] which is solved using a stochastic numerical method [18]. The model describes particles as aggregates composed of primary particles 
which are in turn composed of individual PAHs, thus containing information on particle size, morphology, and the internal structure of the particles. These particles undergo coagulation, sintering and particle rounding due to condensation and surface growth. This is the PAH-primary particle (PAHPP) model [19]. The KMC-aromatic site (KMC-ARS) model tracks each of the PAHs in the particles which evolve according to a list of jump processes [20, 21] which make use of the steady-state assumption [22] for all intermediate species to reduce the computational expense of the simulations. This particular modelling approach has been use to simulate soot formation in laminar flames (see, e.g., ref. [17-19, 23, 24]) and engines (see, e.g., ref. [25]). The KMC-ARS model is unable to predict curvature because it does not allow the incorporation of 5-member rings and it only tracks the coordinates of the carbon atoms which describe the edge of the PAH. But the Gauss-Bonnet theorem [26, 27] which relates the integral of the Gauss curvature over a compact surface, i.e. its total Gauss curvature, with an integer naturally associated to it, i.e. its Euler characteristic, may be of help to us.

The purpose of this paper is to extend the PAH-PP/KMC-ARS model such that we can not only track PAHs but also allow the inclusion of 5member rings and to track the associated change in curvature using the Gauss-Bonnet theorem. Computed fringe length distributions are also compared with experimental measurements [6]. As fringe length is thought to be related to PAH size, these comparisons allow us to investigate the minimum PAH sizes required for inception and condensation which may be different. The influence of different fuels is also briefly studied. First, the flame chemistry and structure are computed using a steady-state burner-stabilised premixed laminar flame code. Second, the particle dynamics is solved using a detailed population balance model where the PAH growth mechanism is extended to allow for the incorporation of 5-member rings which leads to PAH curvature.

\section{Computational method}

The computational method consists of two parts. In the first part, species profiles are computed using a modified version of PREMIX [28], including calculation of the source terms by the method of moments with interpolative closure (MOMIC) using the code published by Revzan et al. [29]. The ABF mechanism, which includes combustion chemistry and the formation 
Table 1: Flame conditions

\begin{tabular}{|c|c|c|c|c|c|c|c|c|}
\hline \multirow[b]{2}{*}{ Flame } & \multicolumn{4}{|c|}{ Unburned gas composition $^{a}$} & \multirow{2}{*}{$\begin{array}{l}\text { Equivalence } \\
\text { ratio }\end{array}$} & \multirow{2}{*}{$\begin{array}{c}\text { Linear velocity }{ }^{b} \\
(\mathrm{~cm} / \mathrm{s})\end{array}$} & \multirow{2}{*}{$\begin{array}{c}\text { Maximum flame } \\
\text { temperature, } T_{\mathrm{m}}(\mathrm{K})\end{array}$} & \multirow{2}{*}{$\begin{array}{c}\text { Burner temperature }{ }^{c} \\
(\mathrm{~K})\end{array}$} \\
\hline & $\mathrm{C}_{2} \mathrm{H}_{4}$ & $\mathrm{C}_{6} \mathrm{H}_{6}$ & $\mathrm{O}_{2}$ & $\mathrm{~N}_{2}$ & & & & \\
\hline $\mathrm{A}$ & 0.444 & & 0.556 & & 2.4 & 4 & 1723 & 333 \\
\hline $\mathrm{B}$ & & 0.053 & 0.199 & 0.748 & 2.0 & 3 & 1713 & 333 \\
\hline
\end{tabular}

and growth pathways of PAHs up to pyrene, and species thermodynamics and transport properties $[30,31]$ are supplied as input. Note that the ABF mechanism includes the mechanism for benzene combustion. The experimental temperature profile $[32,33]$ is imposed to ensure full agreement between the experimental temperature and that seen by the soot model. The transport equations of the moments of the particle size distribution (PSD) are solved to account approximately for the production and consumption of key gas-phase species due to inception, condensation, surface growth and oxidation. A total of six moments, including the zeroth moment, are solved using MOMIC to close the moment transport equations. In the second part, a detailed population balance model is applied as a post-processing step where the imposed temperature and computed species profiles from PREMIX are supplied as input.

We investigate two different flames and the flame conditions are summarised in Table 1. These are atmospheric pressure sooting flames where the main difference is that Flame A [32] is an ethylene flame $(\phi=2.4)$ while Flame B [33] is a benzene flame $(\phi=2.0)$. PREMIX simulations are performed using windward differencing and considering multicomponent transport and thermal diffusion. About 150 grid points are found to be sufficient for convergence.

\section{Detailed population balance model}

A detailed population balance model [17] is used to model soot formation by postprocessing the PREMIX simulations of the burner-stabilized laminar premixed flame. The growth of PAH species within the model is described by a kinetic Monte Carlo-aromatic site (KMC-ARS) model [20], starting from pyrene. The dynamics of the soot particle population is described by the Smoluchowski coagulation equation with additional terms for particle inception, condensation, sintering and surface growth. A brief description 
of the most important aspects of the particle model is given below. Further details may be found elsewhere [17, 19, 20].

In the model, soot particles are represented as aggregates composed of primary particles, where each primary particle is composed of a number of PAHs [19]. The edge of each PAH is described by a list of contiguous site types. These elementary sites include free-edge, zig-zag, armchair and bay sites $[20,34,35]$. This representation allows the exact structure of each individual PAH to be resolved. A primary particle is represented as a set of two or more PAHs. An aggregate is represented as a set of two or more primary particles. Each aggregate stores a list of neighbouring primary particles and resolves the common surface area between each pair of neighbours, where each pair of neighbours can be in point contact, can be fully sintered or can be anywhere in between [19]. The degree of sintering is described by a sintering level, $s \in[0,1][36]$. A sintering level of 0 corresponds to point contact and a sintering level of 1 corresponds to completely sintered.

There are five different particle processes in the model:

Inception A primary particle is formed when two PAH molecules stick following a collision. The rate of collision is determined by a transition regime coagulation kernel [37] which is dependent on the mass and collision diameter of each collision partner [23]. The sticking probability of these two PAHs is determined by a simple collision efficiency model [17]. If the number of 6 -member aromatic rings in each of the collision partners exceeds 16 then they will stick, which provided the best agreement between experimentally derived and computed optical band gap [24]. This implies a unit sticking probability.

Coagulation An aggregate is formed when two (primary or aggregate) particles stick following a collision. The rate of collision is calculated as per the transition regime coagulation kernel. After a coagulation event, two primary particles (one from each collision partner) are assumed to be in point contact. These primaries may undergo subsequent particle rounding due to mass addition [19] via surface growth and condensation, and due to sintering [36].

Surface growth PAHs in a primary particle may grow via surface reactions with gas-phase species. The rate of surface growth is a function of the structure of the PAH and is described by the KMC-ARS model. Two parameters are introduced to differentiate the rate of growth of PAHs 
in a primary particle versus those in the gas phase: the growth factor, $g$, and the critical number of PAHs in a primary particle, $n_{\text {crit }}$. The growth factor $g \in[0,1][19]$ is a multiplier that is applied to the growth rate of PAHs within primary particles once the number of PAHs $\left(n_{\mathrm{PAHs}}\right)$ exceeds $n_{\text {crit }}$. It is intended to account for the possibility that PAHs in large primary particles grow more slowly than PAHs in the gas-phase. We assume $n_{\text {crit }}=4$ because Totton et al. [38] showed that the average stack size for clusters of coronene molecules is around 4. Reactions take place on the edge of a molecule and the edges of PAHs in stacks are exposed to the chemical environment.

Surface growth increases the mass of a PAH, which results in an increase in the sphericity of the primary particle containing the PAH and any neighbouring primary particles [19]. This particle rounding takes the form of an increase in the common surface area between the affected primary particles. The rate of particle rounding is parameterised by a smoothing factor $\sigma \in[0,2][19]$ that relates the change of the common surface area to the change of the volume of a primary particle. A smoothing factor of 0 implies instantaneous coalescence, whereas a smoothing factor of 2 corresponds to no rounding. Consistent with the inception process, if a surface reaction reduces the number of 6 -member aromatic rings in a PAH in a particle (defined as $n_{\mathrm{PAHs}} \geq n_{\text {crit }}$ ) below 16 , the PAH is removed.

Whitesides and Frenklach [13] presented a detailed KMC-MD model of graphene-edge growth with a total of 42 surface transformations. One of the most interesting among them is capping which is the addition of acetylene to an embedded 5-member ring which causes a PAH to curve [39]:

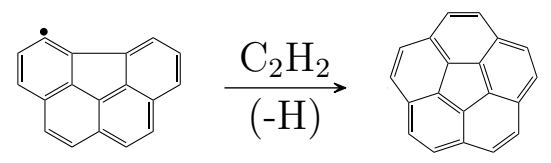

In this paper, hydrogen abstraction and the subsequent capping are represented as a jump process and is added to the KMC-ARS model. A complete list of jump processes, their steady-state rate expressions and the corresponding elementary reaction rate coefficients in the KMCARS model are given in the Supplementary material (Tables S1 and S2). 
The rate coefficient of reaction 1 is taken from the work of You et al. [15] who derived the coefficient using quantum-chemical calculations and reaction rate analysis. The coefficient has an order-of-magnitude accuracy. This coefficient is essential to predicting the capping of an embedded 5-member ring. We find that a rate coefficient estimated based on an analogous acetylene addition and cyclisation on a zig-zag site $[39,40]$ is about two orders of magnitude lower resulting in embedded 5-member rings migrating to adjacent zig-zag sites (jump process S17) and subsequently desorbing (jump process S6) instead of being capped.

By describing the edge of each PAH through a list of site types alone, curved PAHs can be accommodated within the KMC-ARS model. However, updating the structure of the $\mathrm{PAH}$ depends on whether any "bridges" are present. The simplest example of a bridge is the $\mathrm{C}-\mathrm{C}$ bond that joins two benzene rings in a biphenyl molecule. At this stage, we are unable to determine on-the-fly the location of any bridges from a list of site types alone. Therefore, we exclude jump processes which create bridges, i.e., phenyl addition and armchair oxidation by $\mathrm{O}_{2}$ and $\mathrm{OH}$. Before the inclusion of reaction 1, phenyl addition constitutes less than $4 \%$ of the total number of jump processes.

Condensation A particle may grow via condensation of a gas-phase PAH, following a collision between the PAH and a primary or aggregate particle. The rate of collision is calculated as per the transition regime coagulation kernel. Consistent with the inception process, if the condensing PAH has 16 or more 6-member aromatic rings, it will stick to the particle. Rounding by mass addition occurs via the same mechanism as described for the surface growth process.

Sintering Neighbouring primary particles may undergo particle rounding via a sintering process $[36,41,42]$. The rate of sintering between each pair of neighbouring primary particles $p_{i}$ and $p_{j}$ is given [36]:

$$
\frac{\Delta C_{i j}}{\Delta t}=-\frac{1}{\tau_{\mathrm{s}}\left(p_{i}, p_{j}\right)}\left(C_{i j}-S_{\mathrm{sph}}\left(p_{i}, p_{j}\right)\right),
$$

where $C_{i j}$ is the common surface area of primary particles $p_{i}$ and $p_{j}$, and $S_{\mathrm{sph}}\left(p_{i}, p_{j}\right)$ is the surface area of a sphere with the same volume as primaries $p_{i}$ and $p_{j}$. The characteristic sintering time is given [43]: 


$$
\tau_{\mathrm{s}}\left(p_{i}, p_{j}\right)=A_{\mathrm{s}} \times d_{\mathrm{sph}}\left(p_{i}, p_{j}\right) \times \exp \left(\frac{E_{\mathrm{s}}}{T}\left(1-\frac{d_{\mathrm{pri}, \mathrm{crit}}}{d_{\mathrm{sph}}\left(p_{i}, p_{j}\right)}\right)\right)
$$

where $A_{\mathrm{s}}$ is the pre-exponential factor, $d_{\mathrm{sph}}\left(p_{i}, p_{j}\right)$ is the diameter of a sphere with the same volume as that of primaries $p_{i}$ and $p_{j}, E_{\mathrm{s}}$ is the activation energy and $d_{\text {pri,crit }}$ is the critical primary particle diameter below which the primaries are assumed to be liquid-like and 'sinter' instantaneously.

The sintering level $s\left(p_{i}, p_{j}\right)$ determines how far primary particles $p_{i}$ and $p_{j}$ have sintered:

$$
s\left(p_{i}, p_{j}\right)=\frac{\left.\left(S_{\mathrm{sph}}\left(p_{i}, p_{j}\right)\right) / C_{i j}\right)-2^{-1 / 3}}{1-2^{-1 / 3}}
$$

where a value of 0 implies that the primary particles are in point contact, while a value of 1 means that the primary particles are fully sintered.

There are many parameters in the model, but the key parameters we investigate in this work are given in Table 2. The range of $\rho$ is based on the variation in density from nascent to mature soot [19], the ranges of $\sigma$ and $g$ represent physical constraints of the model, and the ranges of $E_{\mathrm{s}}$ and $d_{\text {pri,crit }}$ are based on previously optimised values to describe the aerosol synthesis of silica nanoparticles [44]. The values of the aforementioned parameters and $A_{\mathrm{s}}$ are the same as ref. [17].

\section{Curvature}

As the KMC-ARS model gives us the number of capped 5-member rings we need to develop a theory which allows us to calculate the curvature of the PAH surface if we want to compare the molecular structure of the soot particles with measured data. The Gauss-Bonnet theorem relates the curvature of a surface to its Euler characteristic, which is a number that describes the structure of a PAH in terms of vertices, edges and faces. Given the number of 5- and 6-member rings in a PAH, we demonstrate how this theorem can be used to estimate the average Gauss curvature (or radius of curvature) of 
Table 2: Model parameters in detailed population balance model.

\begin{tabular}{|c|c|c|}
\hline Parameter & Range & Value \\
\hline $\begin{array}{l}\text { 1) Minimum number of } 6 \text {-member } \\
\text { aromatic rings in a PAH for } \\
\text { inception }\end{array}$ & - & 16 \\
\hline $\begin{array}{l}\text { 2) Minimum number of } 6 \text {-member } \\
\text { aromatic rings in a PAH for } \\
\text { condensation }\end{array}$ & - & 16 \\
\hline $\begin{array}{l}\text { 3) Minimum number of } 6 \text {-member } \\
\text { aromatic rings in a PAH in a } \\
\text { particle }\left(n_{\mathrm{PAHs}} \geq n_{\text {crit }}\right) \text { below } \\
\text { which it is removed }\end{array}$ & - & 16 \\
\hline 4) Soot density, $\rho$ & $1 \mathrm{~g} \mathrm{~cm}^{-3} \leq \rho \leq 2 \mathrm{~g} \mathrm{~cm}^{-3}$ & $1.88 \mathrm{~g} \mathrm{~cm}^{-3}$ \\
\hline 5) Smoothing factor, $\sigma$ & $0 \leq \sigma \leq 2$ & 1.69 \\
\hline 6) Growth factor, $g$ & $0 \leq g \leq 1$ & 0.0263 \\
\hline $\begin{array}{l}\text { 7) Critical number of PAHs in a } \\
\text { primary particle before the } \\
\text { growth factor is applied, } n_{\text {crit }}\end{array}$ & $\geq 2$ & 4 \\
\hline \multicolumn{3}{|l|}{ 8) Sintering model: } \\
\hline$-A_{\mathrm{s}}$ & - & $1.1 \times 10^{-14} \mathrm{~s} \mathrm{~m}^{-1}$ \\
\hline$-E_{\mathrm{s}}$ & $1.8 \times 10^{4} \mathrm{~K} \leq E_{\mathrm{s}} \leq 1.8 \times 10^{5} \mathrm{~K}$ & $9.61 \times 10^{4} \mathrm{~K}$ \\
\hline$-d_{\text {pri,crit }}$ & $1 \mathrm{~nm} \leq d_{\text {pri,crit }} \leq 5 \mathrm{~nm}$ & $1.58 \mathrm{~nm}$ \\
\hline
\end{tabular}

the PAH, independent of where the 5-member ring is embedded within the $\mathrm{PAH}$.

Consider a PAH molecule comprising $P$ pentagons and $H$ hexagons. Associate with each interior atom a curvature $\kappa_{P P P}, \kappa_{P P H}, \kappa_{P H H}$ or $\kappa_{H H H}$ depending on whether the atom is part of three pentagons, two pentagons and a hexagon, one pentagon and two hexagons, or three hexagons. The different configurations are illustrated in Fig. 1.

[Figure 1 about here.]

The probabilities that an interior point selected at random will be each of these types are:

$$
\mathbb{P}_{P P P}=F(3) ; \quad \mathbb{P}_{P P H}=F(2) ; \quad \mathbb{P}_{P H H}=F(1) ; \quad \mathbb{P}_{H H H}=F(0) ;
$$


where $F$ is the hypergeometric distribution

$$
F(p)=\frac{\left(\begin{array}{c}
P \\
p
\end{array}\right)\left(\begin{array}{c}
H \\
3-p
\end{array}\right)}{\left(\begin{array}{c}
P+H \\
3
\end{array}\right)},
$$

parameterised by $p$ which is the number of pentagons an interior atom is part of. As a rule of thumb the hypergeometric distribution should be considered when the sample size $(=3)$ is larger than approximately $10 \%$ of the population [45], or 30 aromatic rings or less. Now assign the average Gauss curvature of the PAH molecule to be

$$
\bar{K}=\kappa_{P P P} \mathbb{P}_{P P P}+\kappa_{P P H} \mathbb{P}_{P P H}+\kappa_{P H H} \mathbb{P}_{P H H}+\kappa_{H H H} \mathbb{P}_{H H H} .
$$

When $P=0$ the molecule is flat and hence $\kappa_{H H H}=0$. The Gauss curvature is defined to be

$$
K=\kappa_{1} \kappa_{2}=\left(R_{1} R_{2}\right)^{-1},
$$

where $\kappa_{1}$ and $\kappa_{2}$ are the principal curvatures, and $R_{1}$ and $R_{2}$ are the principal radii of curvature. Figure 2 illustrates the concept of principal curvatures and radii of curvatures at a given point along a surface.

[Figure 2 about here.]

$X_{1}$ and $X_{2}$ are unit vectors tangent to the surface pointing in the direction along which the maximum and minimum curvatures $\kappa_{1}$ and $\kappa_{2}$ are found, hence, the name principal curvatures. The inverse of these curvatures are the principal radii of curvature and can be understood as the radius of a circle that best approximates the curve at that point. $N$ is the unit vector normal to the surface. We will require that Eq. (7) hold for three arrangements of pentagons and hexagons that allow us easy computation of the average Gauss curvature. The Gauss-Bonnet theorem for a closed surface is:

$$
2 \pi \chi=\int_{M} K \mathrm{~d} S=\bar{K} \int_{M} \mathrm{~d} S=\bar{K} S
$$

where $\chi$ is the Euler characteristic, $M$ is any two-dimensional Riemannian manifold, $\mathrm{d} S$ is the area element of the surface and $S$ is the total surface area. We will apply this when $M$ is a closed surface and $\chi=2$ (for a topological sphere). In particular we will consider the smallest possible fullerene, the buckminsterfullerene and the rugbyballene which consist of 20, 60 and 70 carbon atoms respectively as shown in Fig. 3. 
[Figure 3 about here.]

In $\mathrm{C}_{20}$ a pentagon is completely surrounded by pentagons, whereas in $\mathrm{C}_{60}$ and $\mathrm{C}_{70}$ a pentagon is completely surrounded by hexagons. This is an application of the configurations in Figs. 1a and 1c respectively. The isolated pentagon rule (IPR) states that a structure in which a pentagon is completely surrounded by hexagons is stable [46] and $\mathrm{C}_{60}$ and $\mathrm{C}_{70}$ conform to this rule. In the case of $\mathrm{C}_{20}$, the necessity of 12 pentagons in a closed shell demonstrates that lower fullerenes that do not obey the IPR may also be stable [46, 47].

Let our hexagons and pentagons have side length $a(=1.395 \AA$, the length of an aromatic $\mathrm{C}-\mathrm{C}$ bond [22]), then the areas of the pentagon and hexagon are

$$
A_{P}=\frac{\left(5\left(5+2 \times 5^{1 / 2}\right)\right)^{1 / 2}}{4} a^{2} \quad \text { and } \quad A_{H}=\frac{3 \times 3^{1 / 2}}{2} a^{2} \text {. }
$$

For $\mathrm{C}_{20} P=12$ and $H=0$, so that $\bar{K}=\kappa_{P P P}$ and from the Gauss-Bonnet theorem we have

$$
\kappa_{P P P}=\frac{\pi}{3 A_{P}} .
$$

Combining the results for the buckminsterfullerene $(P=12$ and $H=20)$ and rugbyballene $(P=12, H=25)$ we get

$$
\begin{aligned}
\kappa_{P P H} & =\frac{992 \pi}{55\left(3 A_{P}+5 A_{H}\right)}-\frac{19684 \pi}{275\left(12 A_{P}+25 A_{H}\right)}-\frac{22 \pi}{225 A_{P}} \\
\kappa_{P H H} & =\frac{11 \pi}{450 A_{P}}-\frac{124 \pi}{15\left(3 A_{P}+5 A_{H}\right)}+\frac{1036 \pi}{25\left(12 A_{P}+25 A_{H}\right)} .
\end{aligned}
$$

In summary, given the number of 5 - and 6-member rings in a $\mathrm{PAH}$, Eqs. (5-7, 10-13) can be used to estimate the average Gauss curvature of each PAH. Assuming that points on the surface are locally spherical (umbilical points), the principal radii of curvature are equal $(K=\bar{K})$ and Eq. (8) can be used to estimate the radius of curvature as $R_{\mathrm{c}}=\bar{K}^{-1 / 2}$.

\section{Results and discussion}

The computed structure of flame A is shown in the left column of Fig. 4.

[Figure 4 about here.] 
It is qualitatively similar to other laminar premixed ethylene flames modelled using a similar gas-phase chemical mechanism [48]. Soot volume fraction measurements show that soot first appears at a height above the burner, $H$, of around $0.2 \mathrm{~cm}$ [32]. This is consistent with the location where fuel and oxidiser are predicted to disappear and where there is a sharp increase in the PAH concentrations. We see a rise-then-fall in the profile of pyrene because of its consumption from particle inception and condensation on soot surfaces. Also shown in Fig. 4 (right column) is the computed structure of flame B. There are two differences which are important for reasons which will be discussed later. First, the maximum temperature, $T_{\mathrm{m}}$, is $10 \mathrm{~K}$ lower than that measured for flame A at $1713 \mathrm{~K}$. Second, the concentration of the $\mathrm{C}_{2} \mathrm{H}_{2}$ growth species is about an order-of-magnitude lower which is expected because the benzene fuel has to be oxidised to form $\mathrm{C}_{2} \mathrm{H}_{2}$ whereas only one hydrogen molecule has to be removed from ethylene [49]. Therefore, we would expect surface growth to be slower in flame B than in flame A. However, we expect soot to be formed more easily in flame $\mathrm{B}$, despite the lower equivalence ratio, because formation of the first aromatic ring is considered to be the first step towards the formation of soot [50].

HRTEM images were collected at several heights above the burner of both flames A and B [6]. Fringe analysis was performed on these images and the radius of curvature was then extracted from the fringes [6]. A limitation of radius of curvature, $R_{\mathrm{c}}$, is that it is unbounded: $R_{\mathrm{c}}$ is infinite for a planar PAH; whereas Gauss curvature, $K$, is bounded between 0 for a planar PAH and around $31 \mathrm{~nm}^{-2}$ for $\mathrm{C}_{20}$, the smallest possible fullerene. For this reason, a comparison between experimental and computed average Gauss curvature for flames A and B is made in Fig. 5.

[Figure 5 about here.]

(Equation (8) is used to convert the experimental radius of curvature to Gauss curvature. For the benefit of the reader the equivalent radius of curvature comparison may be found in the Supplementary material.) The Gauss curvature of $\mathrm{C}_{20}$ and the well-known buckminsterfullerene $\left(K \sim 9 \mathrm{~nm}^{-2}\right)$ are also shown. Experimentally, the average radius of curvature necessarily excludes "nearly" straight PAHs which would skew the average towards the straight PAHs. Therefore, the Gauss curvature is calculated as an average across PAHs in particles where at least one 5-member ring has been embedded and subsequently capped. Numerical simulation yields satisfactory results when compared to the experimentally determined Gauss curvature, 
in fact, the average Gauss curvature between the two flames are similar as will be discussed later. Given limited measurements of radius of curvature in the literature, one possible explanation for the underprediction is missing reactions which embed a 5-member ring and/or cap an embedded 5-member ring. This is an area of ongoing investigation and we make no attempt here of addressing this issue.

For these simulations we assume that only PAHs with 16 or more aromatic rings are able to stick because, in previous work [24], it provided the best fit against optical band gap derived from extinction measurements [51]. (Optical band gap can be correlated with the number of aromatic rings in PAHs [52].) We find that assuming pyrene dimerisation as the inception step results in no curved PAHs because PAHs do not have sufficient time to grow and incorporate 5-member rings before they form a particle where growth is essentially frozen (growth factor, $g=0.0263$ ). Note that the approach used in this work to compute Gauss curvature is valid even for the very flat molecules that are observed because as long as the number of pentagons and hexagons is approximately less than 30, a very flat molecule would have a correspondingly small Gauss curvature.

To better understand the computed Gauss curvature results, the underlying raw data should be informative. Figure 6 shows the distribution of the fraction of embedded 5-member rings which are capped in PAHs in soot for flame $\mathrm{A}$ at $H=13 \mathrm{~mm}$ (top panel) and for flame $\mathrm{B}$ at $H=11 \mathrm{~mm}$ (bottom panel).

[Figure 6 about here.]

Note that the length of the bars in each pattern represents the fraction of embedded 5-member rings which are capped as opposed to the fraction of PAHs. Both distributions show that there is a large concentration around PAHs which contain 16 6-member aromatic rings - a direct result of the inception/condensation threshold chosen (see parameters 1 and 2 in Table 2) which explains why the average Gauss curvature in Fig. 5 for flames A and $\mathrm{B}$ are similar. Due to the higher temperature and concentration of the $\mathrm{C}_{2} \mathrm{H}_{2}$ growth species in flame $\mathrm{A}$ as compared to flame B, PAHs in flame A tend to be larger and incorporate up to three 5-member rings. Another point of comparison is the percentage of fringes which are curved which is found to be about $19 \%$ and $16 \%$ for flames $\mathrm{A}$ and B, respectively. This is an underestimation of the experimental measurement of around $75 \%$; however, this 
is to be expected given the underestimation of the Gauss curvature that was observed earlier.

As an independent means of assessing the model's ability to predict curvature, we optimise the structures of two representative PAHs predicted by the model in a post-analysis step and make a qualitative comparison with their respective Gauss curvatures. Geometry optimisation is performed using the MM2 [53] minimisation tool in Chem3D 15.1 [54]. While the MM3 potential is known to predict equilibrium bond lengths for fullerenes significantly better than MM2 [55], the optimisation is only performed for illustrative purposes therefore MM2 is deemed to be sufficient for these purposes. By tracking the evolution of the list of site types which describes the edge of each PAH as it undergoes surface growth, we are able to determine the exact structure of both PAHs. Figure 7 shows the most curved structure for flames A and B and the corresponding Gauss curvatures are $3.91 \mathrm{~nm}^{-2}$ (16 hexagons and 3 pentagons) and $2.36 \mathrm{~nm}^{-2}$ (16 hexagons and 2 pentagons).

[Figure 7 about here.]

The structure from flame A has a higher Gauss curvature and its optimised structure does indeed look more curved than the structure from flame B, which provides some reassurance of the validity of the model.

A final note should be made about the Gauss curvature correlation derived in Section 4. The correlation is based upon the interpolation of the curvatures of different fullerenes. (At any point along the surface of a fullerene it has a non-negative curvature.) Therefore, implicit in the application of the correlation is the assumption that the PAH structure is either flat or bends in the same direction. Evidence of negative curvature (a saddle point or imagine a pringle-like surface) has occasionally been observed, for example, at the transition between conical and tubular sections of soot collected from a laminar benzene premixed flame [5] and is attributed to the presence of 7-member aromatic rings [56]. However, the structures identified within soot particles using HRTEM are either nearly straight or exhibit a positive overall curvature [6]. Application of the Gauss curvature correlation to the PAH structures predicted by the KMC-ARS model is also consistent because the KMC-ARS model only allows for the formation of 5- and 6-member aromatic rings.

Fringe length distributions are useful as they have been used to provide insight to the molecules that are available for particle nucleation and surface 
growth [7]. Figure 8 (left column) shows experimental fringe length distributions at two heights above burner representing nascent and mature soot for flames A and B.

[Figure 8 about here.]

It was initially surprising that the distributions were relatively independent of fuel and maturity of the soot particles; however, similar results were found by Botero et al. [8], albeit for a laminar diffusion flame, where the average fringe length measured across a range of fuels and positions in the flame were practically indistinguishable.

Also shown in Fig. 8 (right column) are the computed fringe length distributions for planar PAHs, that is, before the inclusion of reaction 1 . This approximation is made because it is not clear how a representative length can be easily extracted from a curved PAH; however, the majority of the computed PAHs are either flat or, as shown in Fig. 5, just slightly curved. We estimate the fringe length of each PAH structure as the arithmetic mean of its length and width [6]. There is a qualitative agreement between model and experiment where larger fringes are present in increasingly smaller quantities, but - more importantly - the distributions appear to relatively independent of fuel or height which is observed experimentally. Quantitatively, however, the distributions differ notably. The smallest predicted fringe length is about $1.1 \mathrm{~nm}$ which corresponds to a PAH with 16 aromatic rings; it is assumed that only PAHs with 16 or more aromatic rings are able to incept or condense. Experimentally, all fringes larger than the size of one aromatic ring are considered [6]; therefore, the experimental distributions begin at about $0.3 \mathrm{~nm}$. One of the steps in fringe analysis is establishing a minimum fringe length to rule out artifacts [57]. This is usually set at the size of benzene [6] or pyrene [57].

Are fringes the size of pyrene, or even benzene, physically reasonable? There have been a number of studies investigating theoretical aspects of PAH dimerisation as the initial step to particle nucleation. Sabbah et al. [58] found that the equilibrium of pyrene dimerisation strongly favours the dissociation of the dimer at high temperature and that much larger PAHs, PAHs the size of circumcoronene, are required to be able to play a role in nucleation [59-62]. A key assumption made, however, is that dimerisation is governed by equilibrium kinetics. The next question that follows is are PAHs smaller than the size required for inception able to condense onto a particle? Laser microprobe mass spectrometry of nascent soot particles collected 
from a laminar ethene diffusion flame shows that masses the size of pyrene are present in significant quantities [63]. Schuetz and Frenklach [64] studied the non-equilibrium dynamics of binary collisions between pyrene molecules and found that development of internal rotors are sufficient to stabilise a pyrene dimer long enough to grow in size due to chemical reactions. While it is argued [59] that it is unclear whether a dimer is able to survive collision with gas-phase molecules as most of these collisions are non-reactive, this hypothesis is plausible for the condensation of a pyrene molecule onto a larger, more stable PAH cluster [61,62]. In order to reconcile experimental and computed soot volume fraction in a opposed-jet ethylene diffusion flame, Wang et al. [65] argued that aromatic species such as benzene and naphthalene may condense readily onto the soot surface. We test the hypothesis that PAHs smaller than the PAH size required for inception (16 rings) are able to condense onto a particle. Figure 9 shows the computed fringe length distribution for flame A at $H=13 \mathrm{~mm}$ for the base case and when the condensation threshold is lowered to 4 rings which goes some way towards reconciling the discrepancy with the experiments.

[Figure 9 about here.]

The results for flame B are similar and a condensation threshold of 4 rings is chosen because pyrene is the gas-phase transfer species. The results suggest that species as small as pyrene could condense successfully onto a particle. It would be of interest to determine whether the model predicts the presence of small fringes on the particle surface or the core of the particle; however, this is beyond the scope of the present paper.

\section{Conclusions}

A detailed population balance model, which includes the KMC-ARS model for detailed PAH growth, is used to model soot formation in laminar premixed ethylene and benzene flames. We demonstrate that the GaussBonnet theorem can be used to predict the Gauss curvature of PAHs in soot. The theorem is used to derive a correlation between the number of 5- and 6-member rings in a PAH and its Gauss curvature, independent of where the 5-member ring is embedded within the PAH structure. As a proof of concept, a single-step transformation which caps embedded 5-member rings in a PAH is added to the KMC-ARS model with the rate coefficient of the capping reaction taken from literature. Alternative pathways will be added 
in future. To accommodate the modelling of curved PAHs, we describe the edge of each PAH through a list of site types alone. There is a satisfactory agreement between the simulated and experimentally determined Gauss curvature reported in the literature. While the average Gauss curvature between the ethylene and benzene flames are similar, the model predicts that larger curved PAHs which incorporate more 5-member rings are formed in the ethylene flame. This may be explained by the higher temperature and computed concentration of the $\mathrm{C}_{2} \mathrm{H}_{2}$ growth species in the ethylene flame as compared with the benzene flame. Since the model resolves the structure of each PAH, we can extract a characteristic length from each PAH which can be compared with experimental fringe length distributions. It is found that assuming that only PAHs with 16 or more aromatic rings are able to dimerise or condense onto a particle leads to an overestimation of the smallest fringe length. Lowering the condensation threshold to pyrene improves the agreement with the experimental measurements. The results suggest that PAHs smaller than the size required for inception are able to condense onto particles.

\section{Acknowledgements}

The authors would like to thank Prof. Dr. Christof Schulz for helpful discussions on the possibility of wave-like PAHs. This research is supported by the National Research Foundation, Prime Minister's Office, Singapore under its CREATE programme. Mr. Edward K. Y. Yapp was supported by the Gates Cambridge, Cambridge, UK. Prof. Markus Kraft was supported by the Weierstrass Institute for Applied Analysis and Stochastics, Berlin, Germany.

\section{References}

[1] H. W. Kroto, J. R. Heath, S. C. O'Brien, R. F. Curl, R. E. Smalley, C60: buckminsterfullerene, Nature 318 (1985) 162-163.

[2] Ph. Gerhardt, S. Löffler, K. H. Homann, Polyhedral carbon ions in hydrocarbon flames, Chem. Phys. Lett. 137 (1987) 306-310.

[3] K.-H. Homann, Fullerenes and soot formation-new pathways to large particles in flames, Angew. Chem. Int. Ed. 37 (1998) 2434-2451. 
[4] M. Frenklach, L. B. Ebert, Comment on the proposed role of spheroidal carbon clusters in soot formation, J. Phys. Chem. 92 (1988) 561-563.

[5] W. J. Grieco, J. B. Howard, L. C. Rainey, J. B. Vander Sande, Fullerenic carbon in combustion-generated soot, Carbon 38 (2000) 597-614.

[6] B. Apicella, P. Pré, M. Alfè, A. Ciajolo, V. Gargiulo, C. Russo, A. Tregrossi, D. Deldique, J. N. Rouzaud, Soot nanostructure evolution in premixed flames by high resolution electron transmission microscopy (HRTEM), Proc. Combust. Inst. 35 (2015) 1895-1902.

[7] P. D. Teini, D. M. A. Karwat, A. Atreya, Observations of nascent soot: molecular deposition and particle morphology, Combust. Flame 158 (2011) 2045-2055.

[8] M. L. Botero, D. Chen, S. González-Calera, D. Jefferson, M. Kraft, HRTEM evaluation of soot particles produced by the non-premixed combustion of liquid fuels, Carbon 96 (2016) 459-473.

[9] M. L. Botero, E. M. Adkins, S. González-Calera, H. Miller, M. Kraft, PAH structure analysis of soot in a non-premixed flame using highresolution transmission electron microscopy and optical band gap analysis, Combust. Flame 164 (2016) 250-258.

[10] A. Violi, A. Kubota, T. N. Truong, W. J. Pitz, C. K. Westbrook, A. F. Sarofim, A fully integrated kinetic Monte Carlo/molecular dynamics approach for the simulation of soot precursor growth, Proc. Combust. Inst. 29 (2002) 2343-2349.

[11] A. Violi, Modeling of soot particle inception in aromatic and aliphatic premixed flames, Combust. Flame 139 (2004) 279-287.

[12] A. Violi, A. F. Sarofim, G. A. Voth, Kinetic Monte Carlo-molecular dynamics approach to model soot inception, Combust. Sci. Technol. 176 (2004) 991-1005.

[13] R. Whitesides, M. Frenklach, Detailed kinetic Monte Carlo simulations of graphene-edge growth, J. Phys. Chem. A 114 (2010) 689-703.

[14] R. Whitesides, M. Frenklach, Effect of reaction kinetics on grapheneedge morphology and composition, Z. Phys. Chem. 229 (2014) 597-614. 
[15] X. You, R. Whitesides, D. Zubarev, W. A. Lester Jr., M. Frenklach, Bay-capping reactions: kinetics and influence on graphene-edge growth, Proc. Combust. Inst. 33 (2011) 685-692.

[16] R. I. Singh, A. M. Mebel, M. Frenklach, Oxidation of graphene-edge six- and five-member rings by molecular oxygen, J. Phys. Chem. A 119 (2015) 7528-7547.

[17] D. Chen, Z. Zainuddin, E. Yapp, J. Akroyd, S. Mosbach, M. Kraft, A fully coupled simulation of PAH and soot growth with a population balance model, Proc. Combust. Inst. 34 (2013) 1827-1835.

[18] M. Balthasar, M. Kraft, A stochastic approach to calculate the particle size distribution function of soot particles in laminar premixed flames, Combust. Flame 133 (2003) 289-298.

[19] M. Sander, R. I. A. Patterson, A. Braumann, A. Raj, M. Kraft, Developing the PAH-PP soot particle model using process informatics and uncertainty propagation, Proc. Combust. Inst. 33 (2011) 675-683.

[20] A. Raj, M. Celnik, R. Shirley, M. Sander, R. Patterson, R. West, M. Kraft, A statistical approach to develop a detailed soot growth model using PAH characteristics, Combust. Flame 156 (2009) 896-913.

[21] A. Raj, P. L. W. Man, T. S. Totton, M. Sander, R. A. Shirley, M. Kraft, New polycyclic aromatic hydrocarbon (PAH) surface processes to improve the model prediction of the composition of combustion-generated PAHs and soot, Carbon 48 (2010) 319-332.

[22] M. Frenklach, H. Wang, Detailed mechanism and modeling of soot particle formation, in: H. Bockhorn (Ed.), Soot Formation in CombustionMechanisms and Models, Springer, Berlin, 1994, pp. 165-190.

[23] E. K. Y. Yapp, D. Chen, J. Akroyd, S. Mosbach, M. Kraft, J. Camacho, H. Wang, Numerical simulation and parametric sensitivity study of particle size distributions in a burner-stabilised stagnation flame, Combust. Flame 162 (2015) 2569-2581.

[24] E. K. Y. Yapp, R. I. A. Patterson, J. Akroyd, S. Mosbach, E. M. Adkins, J. H. Miller, M. Kraft, Numerical simulation and parametric sensitivity 
study of optical band gap in a laminar co-flow ethylene diffusion flame, Combust. Flame 167 (2016) 320-334.

[25] S. Mosbach, M. S. Celnik, A. Raj, M. Kraft, H. R. Zhang, S. Kubo, K.O. Kim, Towards a detailed soot model for internal combustion engines, Combust. Flame 156 (2009) 1156-1165.

[26] C. F. Gauss, Disquisitiones generales circas superficies curvas, Comment. Soc. Reg. Sci. Göttingen. Rec. 6 (1828).

[27] O. Bonnet, Mémoire sur la théorie générale des surfaces, J. de l'École Polytechnique 19 (1848) 1-146.

[28] R. J. Kee, J. F. Grcar, M. D. Smooke, J. A. Miller, PREMIX: a FORTRAN program for modeling steady laminar one-dimensional premixed flames, Report No. SAND85-8240, Sandia National Laboratories, Livermore, CA, USA, 1985.

[29] K. L. Revzan, N. J. Brown, M. Frenklach, http://www.me. berkeley.edu/soot. Accessed: 21 April 2016.

[30] J. Appel, H. Bockhorn, M. Frenklach, Kinetic modeling of soot formation with detailed chemistry and physics: laminar premixed flames of $\mathrm{C}_{2}$ hydrocarbons, Combust. Flame 121 (2000) 122-136.

[31] H. Wang, M. Frenklach, A detailed kinetic modeling study of aromatics formation in laminar premixed acetylene and ethylene flames, Combust. Flame 110 (1997) 173-221.

[32] A. Ciajolo, A. D'Anna, R. Barbella, A. Tregrossi, A. Violi, The effect of temperature on soot inception in premixed ethylene flames, Symp. (Int.) Combust. 26 (1996) 2327-2333.

[33] C. Russo, F. Stanzione, A. Tregrossi, M. Alfè, A. Ciajolo, The effect of temperature on the condensed phases formed in fuel-rich premixed benzene flames, Combust. Flame 159 (2012) 2233-2242.

[34] M. Celnik, A. Raj, R. West, R. Patterson, M. Kraft, Aromatic site description of soot particles, Combust. Flame 155 (2008) 161-180.

[35] M. Frenklach, On surface growth mechanism of soot particles, Symp. (Int.) Combust. 26 (1996) 2285-2293. 
[36] M. Sander, R. H. West, M. S. Celnik, M. Kraft, A detailed model for the sintering of polydispersed nanoparticle agglomerates, Aerosol Sci. Technol. 43 (2009) 978-989.

[37] R. I. A. Patterson, J. Singh, M. Balthasar, M. Kraft, W. Wagner, Extending stochastic soot simulation to higher pressures, Combust. Flame 145 (2006) 638-642.

[38] T. S. Totton, D. Chakrabarti, A. J. Misquitta, M. Sander, D. J. Wales, M. Kraft, Modelling the internal structure of nascent soot particles, Combust. Flame 157 (2010) 909-914.

[39] M. Frenklach, C. A. Schuetz, J. Ping, Migration mechanism of aromaticedge growth, Proc. Combust. Inst. 30 (2005) 1389-1396.

[40] R. Whitesides, D. Domin, R. Salomón-Ferrer, W. A. Lester Jr., M. Frenklach, Embedded-ring migration on graphene zigzag edge, Proc. Combust. Inst. 32 (2009) 577-583.

[41] R. H. West, R. A. Shirley, M. Kraft, C. F. Goldsmith, W. H. Green, A detailed kinetic model for combustion synthesis of titania from $\mathrm{TiCl}_{4}$, Combust. Flame 156 (2009) 1764-1770.

[42] W. J. Menz, M. Kraft, A new model for silicon nanoparticle synthesis, Combust. Flame 160 (2013) 947-958.

[43] S. Tsantilis, H. Briesen, S. E. Pratsinis, Sintering time for silica particle growth, Aerosol Sci. Technol. 34 (2001) 237-246.

[44] S. Shekar, A. J. Smith, W. J. Menz, M. Sander, M. Kraft, A multidimensional population balance model to describe the aerosol synthesis of silica nanoparticles, J. Aerosol Sci. 44 (2012) 83-98.

[45] F. W. Breyfogle III, Implementing six sigma: smarter solutions using statistical methods, John Wiley \& Sons, New Jersey, USA, 2003.

[46] $\mathrm{H}$. W. Kroto, The stability of the fullerenes $\mathrm{C}_{n}$, with $n=24,28,32$, 36, 50, 60 and 70, Nature 329 (1987) 529-531.

[47] F. Lin, E. S. Sørensen, C. Kallin, A. J. Berlinsky, $\mathrm{C}_{20}$, the smallest fullerene, in K. D. Sattler (Ed.), Handbook of nanophysics. Clusters and fullerenes, CRC Press, Boca Raton, 2010, pp. 1-11. 
[48] H. Wang, B. Zhao, B. Wyslouzil, K. Streletzky, Small-angle neutron scattering of soot formed in laminar premixed ethylene flames, Proc. Combust. Inst. 29 (2002) 2749-2757.

[49] F. Battin-Leclerc, Detailed chemical kinetic models for the lowtemperature combustion of hydrocarbons with application to gasoline and diesel fuel surrogates, Prog. Energy Combust. Sci. 34 (2008) 440498.

[50] M. Frenklach, H. Wang, Detailed modeling of soot particle nucleation and growth, Symp. (Int.) Combust. 23 (1990) 1559-1566.

[51] E. M. Adkins, J. H. Miller, Extinction measurements for optical band gap determination of soot in a series of nitrogen-diluted ethylene/air non-premixed flames, Phys. Chem. Chem. Phys. 17 (2015) 2686-2695.

[52] J. Robertson, E. P. O'Reilly, Electronic and atomic structure of amorphous carbon, Phys. Rev. B 35 (1987) 2946-2957.

[53] N. L. Allinger, Y. H. Yuh, J.-H. Lii, Molecular mechanics. The MM3 force field for hydrocarbons. 1, J. Am. Chem. Soc. 111 (1989) 8551-8566.

[54] J. J. P. Stewart, Quantum Chemistry Exchange Program No. 455, Indiana University, Bloomington, IN, 1983; CS Chem3D 15.1, CambridgeSoft, Cambridge, MA, 2016.

[55] R. L. Murry, J. R. Colt, G. E. Scuseria, How accurate are molecular mechanics predictions for fullerenes? A benchmark comparison with Hartree-Fock self-consistent field results, J. Phys. Chem. 97 (1993) 49544959.

[56] S. Lijima, T. Ichihashi, Y. Ando, Pentagons, heptagons and negative curvature in graphite microtubule growth, Nature 356 (1992) 776-778.

[57] R. L. Vander Wal, A. J. Tomasek, Soot oxidation: dependence upon initial nanostructure, Combust. Flame 134 (2003) 1-9.

[58] H. Sabbah, L. Biennier, S. J. Klippenstein, I. R. Sims, B. R. Rowe, Exploring the role of PAHs in the formation of soot: pyrene dimerization, J. Phys. Chem. Lett. 1 (2010) 2962-2967. 
[59] H. Wang, Formation of nascent soot and other condensed-phase materials in flames, Proc. Combust. Inst. 33 (2011) 41-67.

[60] T. S. Totton, A. J. Misquitta, M. Kraft, A quantitative study of the clustering of polycyclic aromatic hydrocarbons at high temperatures, Phys. Chem. Chem. Phys. 14 (2012) 4081-4094.

[61] D. Chen, T. S. Totton, J. W. J. Akroyd, S. Mosbach, M. Kraft, Sizedependent melting of polycyclic aromatic hydrocarbon nano-clusters: a molecular dynamics study, Carbon 67 (2014) 79-91.

[62] D. Chen, J. Akroyd, S. Mosbach, D. Opalka, M. Kraft, Solid-liquid transitions in homogenous ovalene, hexabenzocoronene and circumcoronene clusters: a molecular dynamics study, Combust. Flame 162 (2015) 486495.

[63] R. A. Dobbins, R. A. Fletcher, H.-C. Chang, The evolution of soot precursor particles in a diffusion flame, Combust. Flame 115 (1998) $285-298$.

[64] C. A. Schuetz, M. Frenklach, Nucleation of soot: molecular dynamics simulations of pyrene dimerization, Proc. Combust. Inst. 29 (2002) $2307-2314$.

[65] H. Wang, D. X. Du, C. J. Sung, C. K. Law, Experiments and numerical simulation on soot formation in opposed-jet ethylene diffusion flames, Symp. (Int.) Combust. 26 (1996) 2359-2368.

[66] K. Crane, F. de Goes, M. Desbrun, P. Schröder, Digital geometry processing with discrete exterior calculus, in: C. Keenan (Ed.), ACM SIGGRAPH 2013 Courses, ACM, New York, 2013, pp. 16-18. 


\section{List of Figures}

1 Different configurations of pentagons and hexagons. An interior atom is part of (a) three pentagons, (b) two pentagons and one hexagon, (c) one pentagon and two hexagons and $(\mathrm{d})$ three hexagons. . . . . . . . . . . . . . . . 26

2 The Gauss curvature is defined as the product of the two principal curvatures $\kappa_{1}$ and $\kappa_{2}$ at any given point along a surface. $X_{1}$ and $X_{2}$ are unit vectors tangent to the surface along which the maximum and minimum curvatures are found and $N$ is the unit vector normal to the surface. Used with permission [66]. . 27

3 Various closed surfaces which display a range of curvatures. . . 28

4 Computed mole fraction profiles of the major gas-phase species and selected aromatic species for flame A (left column) and flame B (right column) and the temperature measured by a silica-coated fine wire $25 \mu \mathrm{m} \mathrm{Pt} / \mathrm{Pt}-13 \% \mathrm{Rh}$ thermocouple (flame A [32] and flame B [33]). . . . . . . . . . . . . . . . . . 29

5 Comparison of the experimental (symbols) and computed (line) average Gauss curvature of PAHs in particles for flame A (left panel) and flame B (right panel). The shaded areas represent \pm one standard deviation in the computed distribution. The horizontal lines represent the Gauss curvature of $\mathrm{C}_{20}\left(K \sim 31 \mathrm{~nm}^{-2}\right)$ and the buckminsterfullerene $\left(K \sim 9 \mathrm{~nm}^{-2}\right) .30$

6 The fraction of embedded 5-member rings capped within PAHs in particles, sorted by the number of 6-member rings in the $\mathrm{PAH}$, for flame $\mathrm{A}$ at $H=13 \mathrm{~mm}$ (top panel) and for flame $\mathrm{B}$ at $H=11 \mathrm{~mm}$ (bottom panel). The length of the bars in each pattern represents the fraction of embedded 5-member rings which are capped. (For interpretation of the references to colour in this figure legend, the reader is referred to the web version of this article.) . . . . . . . . . . . . . 31

7 Representative structures predicted by the model and optimised using Chem3D 15.1 [54]. . . . . . . . . . . . . . 32

8 Comparison of experimental [6] (left column) and computed (right column) fringe length distributions at two heights above the burner representing nascent (unfilled) and mature (filled) soot for flames A (top row) and B (bottom row). . . . . . . 33 
9 Sensitivity of the computed fringe length distributions to the condensation threshold for flame A at $H=13 \mathrm{~mm}$. . . . . . . 34 


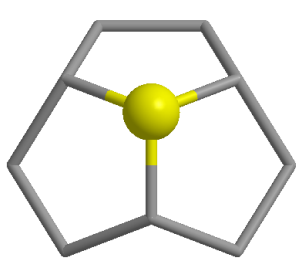

a

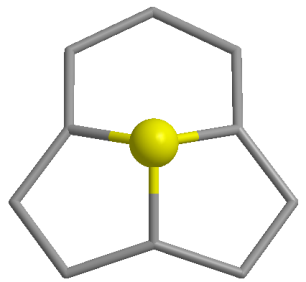

$\mathrm{b}$

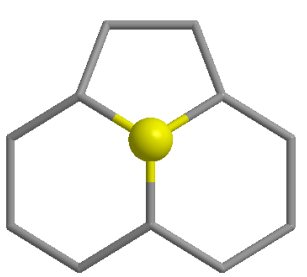

c

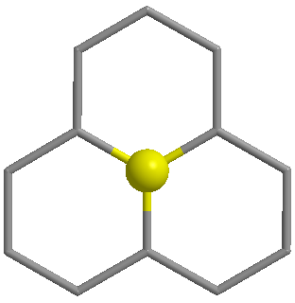

d

Figure 1: Different configurations of pentagons and hexagons. An interior atom is part of (a) three pentagons, (b) two pentagons and one hexagon, (c) one pentagon and two hexagons and (d) three hexagons. 


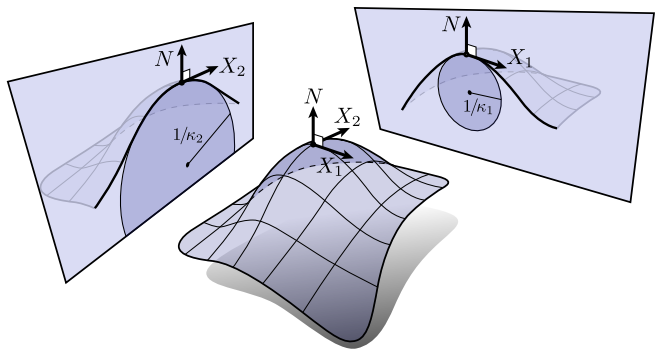

Figure 2: The Gauss curvature is defined as the product of the two principal curvatures $\kappa_{1}$ and $\kappa_{2}$ at any given point along a surface. $X_{1}$ and $X_{2}$ are unit vectors tangent to the surface along which the maximum and minimum curvatures are found and $N$ is the unit vector normal to the surface. Used with permission [66]. 


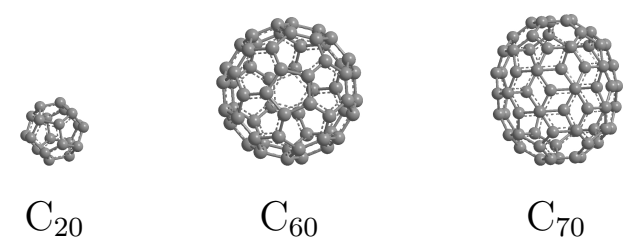

Figure 3: Various closed surfaces which display a range of curvatures. 

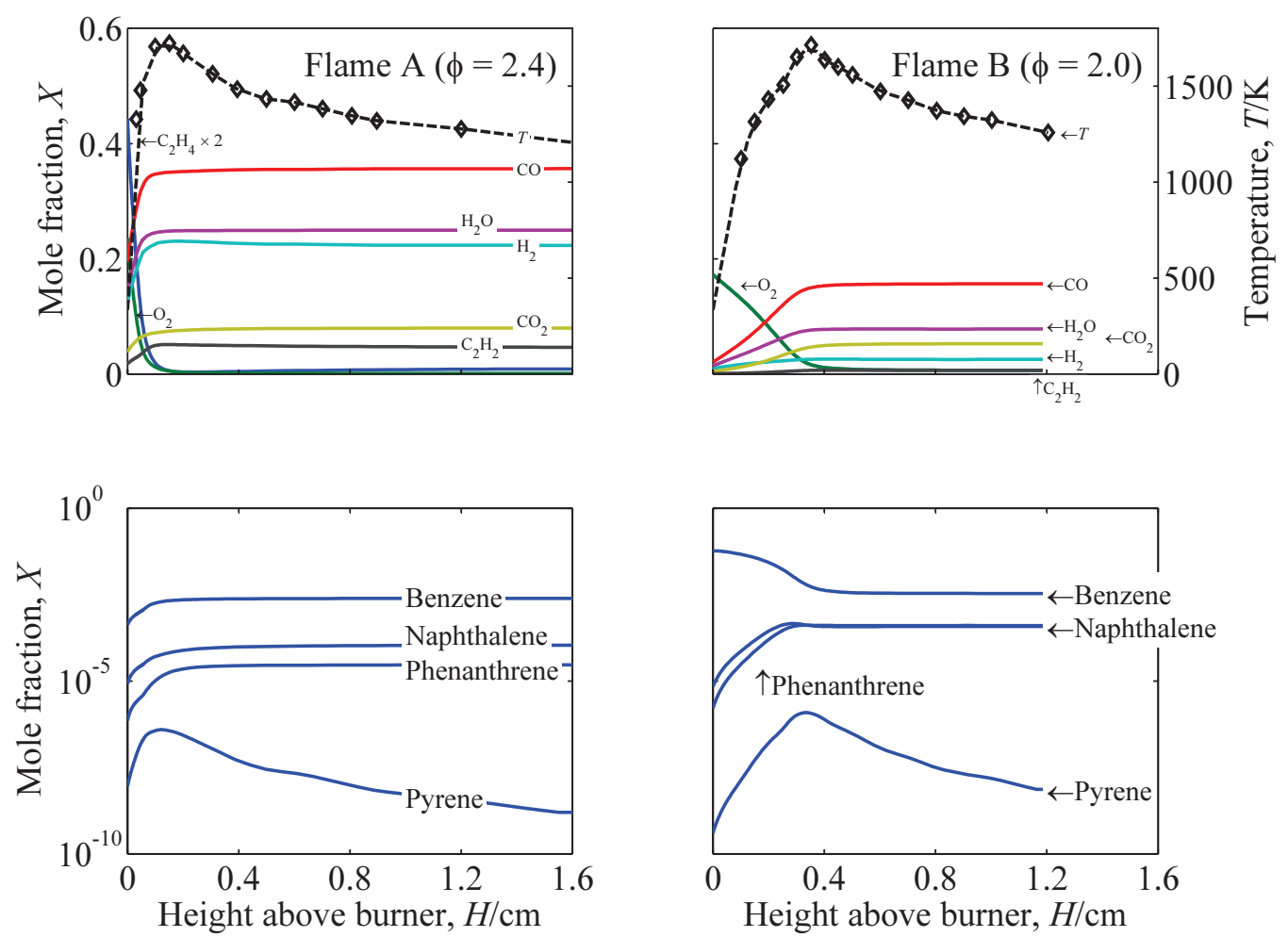

Figure 4: Computed mole fraction profiles of the major gas-phase species and selected aromatic species for flame A (left column) and flame B (right column) and the temperature measured by a silica-coated fine wire $25 \mu \mathrm{m} \mathrm{Pt} / \mathrm{Pt}-13 \% \mathrm{Rh}$ thermocouple (flame A [32] and flame B [33]). 

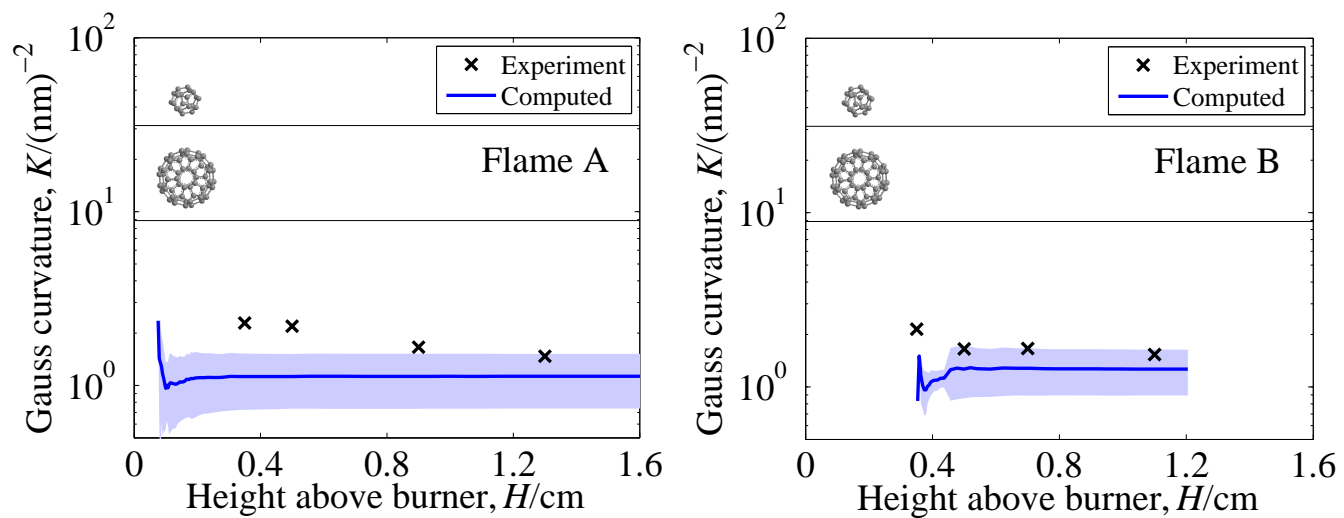

Figure 5: Comparison of the experimental (symbols) and computed (line) average Gauss curvature of PAHs in particles for flame A (left panel) and flame B (right panel). The shaded areas represent \pm one standard deviation in the computed distribution. The horizontal lines represent the Gauss curvature of $\mathrm{C}_{20}\left(K \sim 31 \mathrm{~nm}^{-2}\right)$ and the buckminsterfullerene $\left(K \sim 9 \mathrm{~nm}^{-2}\right)$. 

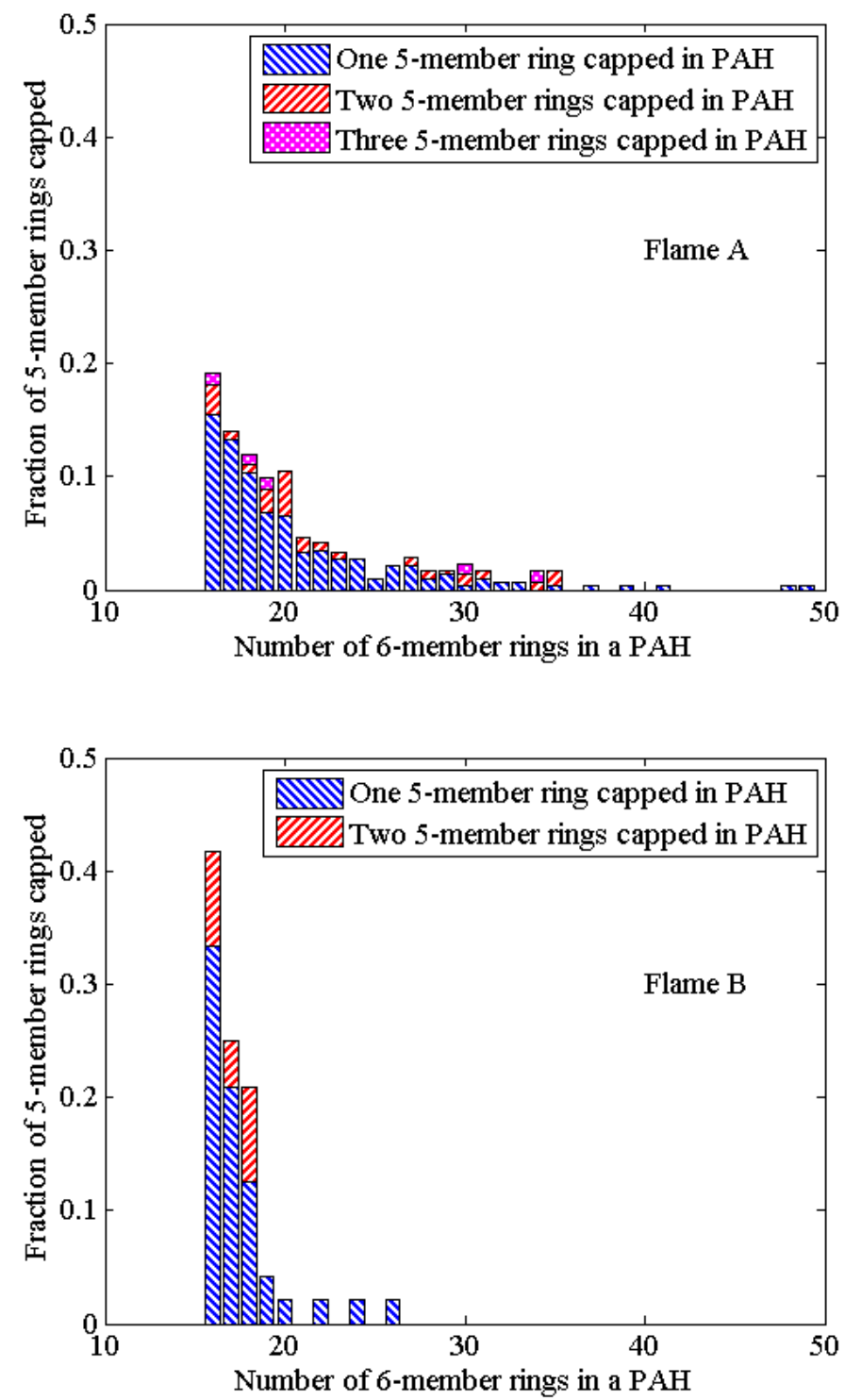

Figure 6: The fraction of embedded 5-member rings capped within PAHs in particles, sorted by the number of 6 -member rings in the PAH, for flame A at $H=13 \mathrm{~mm}$ (top panel) and for flame $\mathrm{B}$ at $H=11 \mathrm{~mm}$ (bottom panel). The length of the bars in each pattern represents the fraction of embedded 5-member rings which are capped. (For interpretation of the references to colour in this figure legend, the reader is referred to the web version of this article.) 


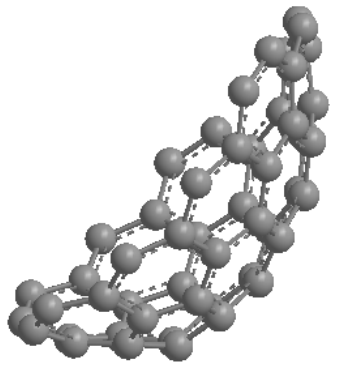

Flame A

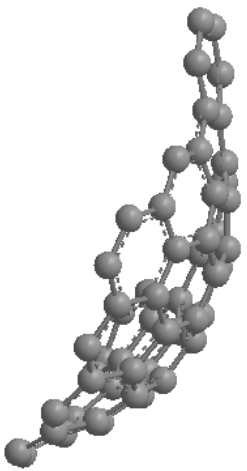

Flame B

Figure 7: Representative structures predicted by the model and optimised using Chem3D 15.1 [54]. 

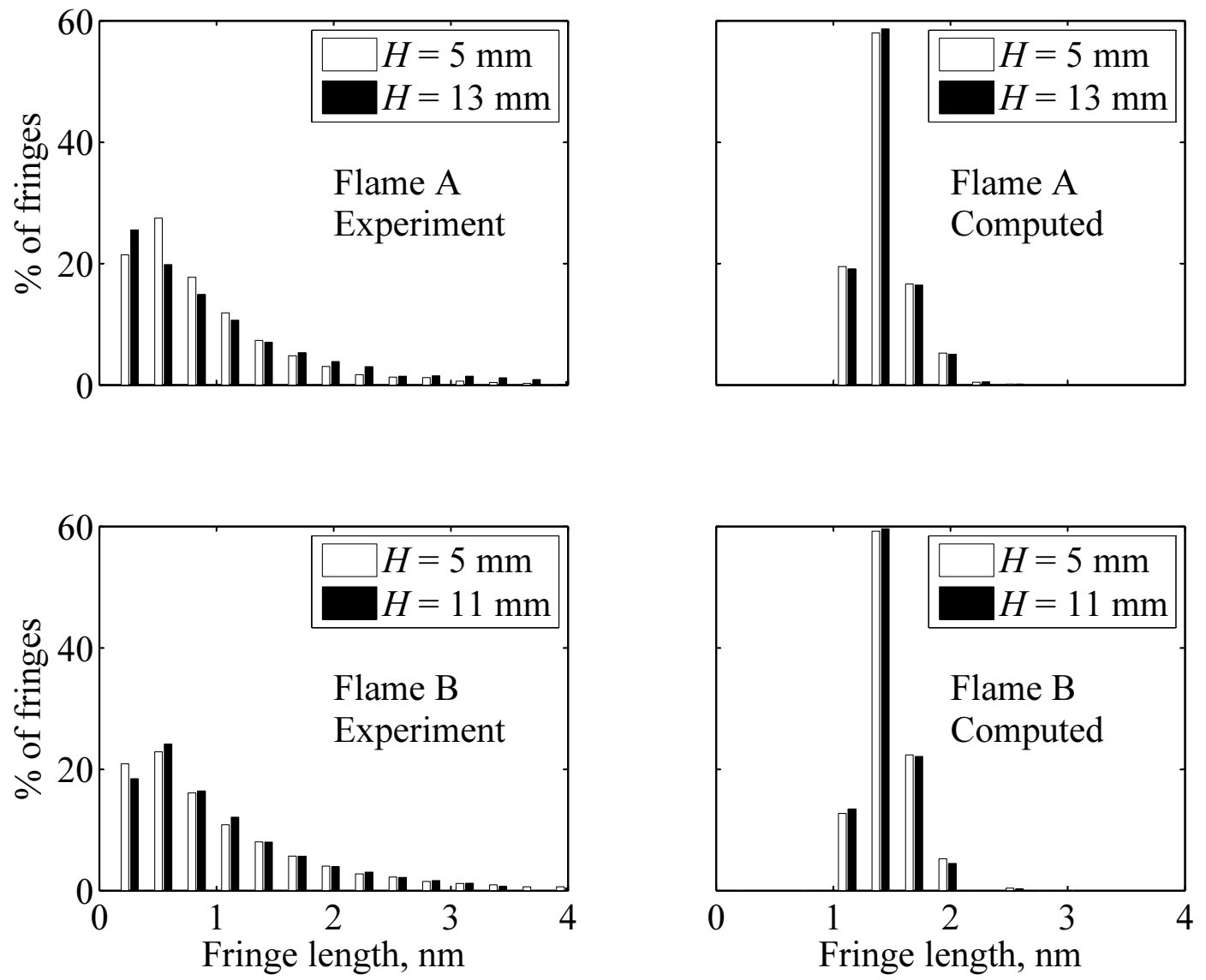

Figure 8: Comparison of experimental [6] (left column) and computed (right column) fringe length distributions at two heights above the burner representing nascent (unfilled) and mature (filled) soot for flames A (top row) and B (bottom row). 


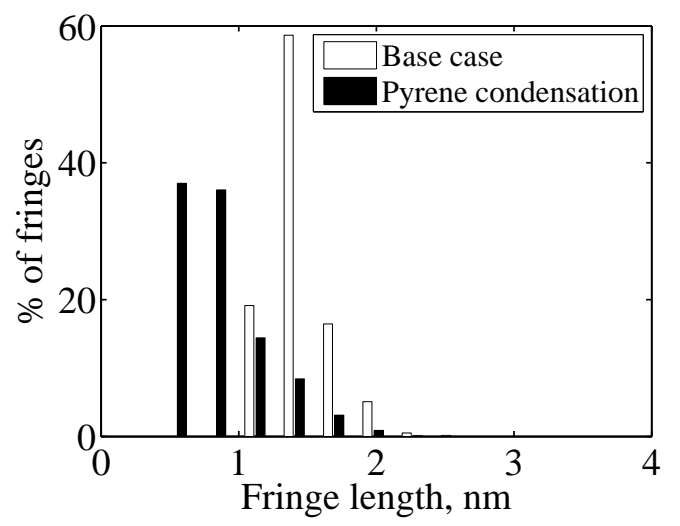

Figure 9: Sensitivity of the computed fringe length distributions to the condensation threshold for flame A at $H=13 \mathrm{~mm}$. 